\title{
A Symptomatic High Riding Jugular Bulb with Diverticulum: An Uncommon Entity
}

\author{
Karthik Marpalli Vasudeva ${ }^{1}$, Kshitij Deependra Wani \\ ${ }^{1}$ Assistant professor, Department of Radiodiagnosis, A.J. Institute of Medical Sciences and Research Centre, Mangalore, \\ Karnataka, 'Junior resident, Department of Radiodiagnosis, A.J. Institute of Medical Sciences and Research Centre, \\ Mangalore, Karnataka, India
}

Corresponding author: Karthik Marpalli Vasudeva, Assistant professor, Department of Radiodiagnosis, A.J. Institute of Medical Sciences and Research Centre, Mangalore, Karnataka, India

DOI: http://dx.doi.org/10.21276/ijcmsr.2020.5.1.56

How to cite this article: Karthik Marpalli Vasudeva, Kshitij Deependra Wani. A symptomatic high riding jugular bulb with diverticulum: an uncommon entity. International Journal of Contemporary Medicine Surgery and Radiology. 2020;5(1):A256-A257.

\section{A B S T R A C T}

Introduction: Jugular bulb anomalies like a high riding jugular bulb or dehiscent jugular bulb are not exactly uncommon in the general population. Most of them are purely incidental in finding because they remain asymptomatic in many of the cases. The radiological reporting of these incidental findings is essential so as to avoid any future medical or medicolegal complications during any of the associated surgeries.

Case report: A 59-year-old lady, with no known co-morbidities, came to the ENT outpatient department of our hospital with complaints of headache for 3 months and continuous tinnitus for 1 month. After an unremarkable clinical examination, the patient was referred to the radiology department to undergo a computed tomography scan of the head to find out the cause of the headache and tinnitus. The computed tomography scan revealed a high riding jugular bulb which extended cranial to the floor of the internal auditory canal with a small diverticulum protruding from it. The patient was managed conservatively.

Conclusion: Jugular Bulb anomalies can present with tinnitus and headache. CT scan is the preferred mode to evaluate these conditions and treatment is generally conservative with surgery reserved for cases with debilitating symptoms.

Keywords: High Riding Jugular Bulb, Jugular Bulb Diverticulum, Sigmoid Plate Dehiscence

\section{INTRODUCTION}

Jugular bulb anomalies like a high riding jugular bulb or dehiscent jugular bulb are not exactly uncommon in the general population. Most of them are purely incidental in finding because they remain asymptomatic in many of the cases. They may present with symptoms of tinnitus and hearing loss. The treatment of choice in these cases is mostly conservative consisting of regular follow up. Surgery is required in a few selective cases where symptoms like hearing loss are debilitating. The radiological reporting of these incidental findings is essential so as to avoid any future medical or medicolegal complications during any of the associated surgeries.

\section{CASE REPORT}

A 59-year-old lady, with no known co-morbidities, came to the ENT outpatient department of our hospital with complaints of headache for 3 months and continuous tinnitus for 1 month. The headache was insidious in onset, diffuse in nature with no predisposing or precipitating factors and showed no diurnal variation. The tinnitus was acute in onset, subjective in nature and the patient complained that it was more on the left side.

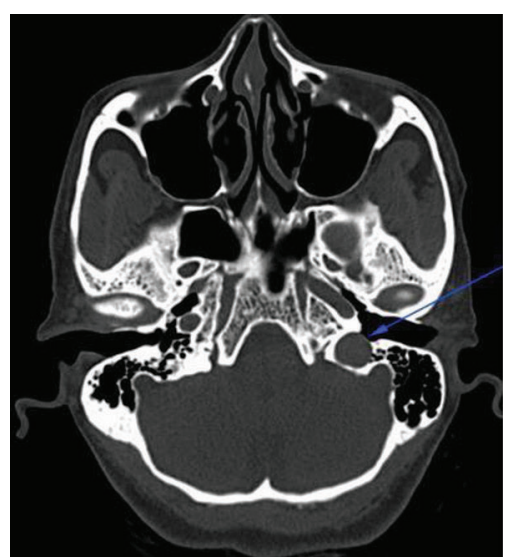

Figure-1: Showing partial dehiscence of the sigmoid plate.

After an unremarkable clinical examination, the patient was referred to the radiology department to undergo a computed tomography scan of the head to find out the cause of the headache and tinnitus. The computed tomography scan revealed a high riding jugular bulb which extended cranial to the floor of the internal auditory canal with a small diverticulum protruding from it. The sigmoid plate was also thinned out. The patient was managed conservatively. 


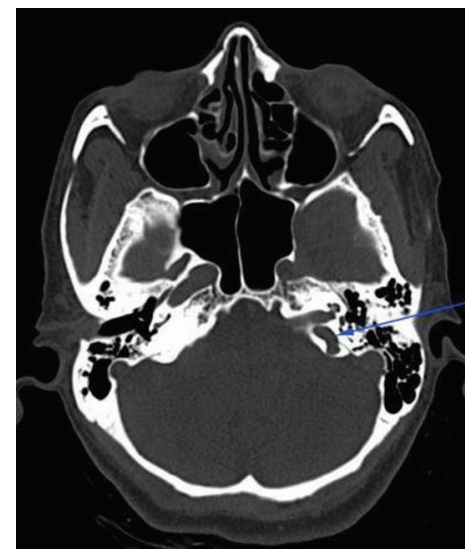

Figure-2: A high riding jugular bulb on the left side.

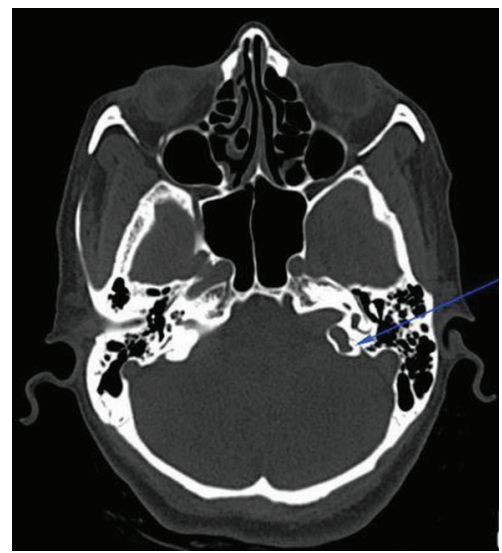

Figure-3: A Jugular bulb diverticulum.

\section{DISCUSSION}

The jugular bulb is the coming together of lateral venous sinuses and is situated in the jugular fossa. As the internal jugular vein moves through the jugular foramen these sinuses drain into it. A thin plate of sigmoid bone separates the jugular bulb from the middle ear cavity. If the bone is too thin the bulb may protrude into the middle ear cavity (Fig 1). A high riding jugular bulb (HRJB) is called so when the most cephalic part of the bulb extends superior to the internal auditory canal or rises to the level of the basal turn of the cochlea. ${ }^{1,2}$

Jugular bulb anomalies like a high riding jugular bulb (HRJB) (Fig. 2) and jugular diverticulum (JD) (Fig. 3) are not particularly uncommon per se. Depending on the mass effect caused by the anomaly on adjacent structures it may cause symptoms like headache, vertigo, and tinnitus and may lead to sensorineural hearing loss if it erodes the wall of the middle ear. The vestibular aqueduct is the most common structure affected in the case of dehiscence. However many of them may remain asymptomatic or show minimal symptoms. While CT is more sensitive to detecting dehiscence as compared to MRI, the volume averaging component of CT scanners may lead to radiologists' "overcalling" or "under calling" the extent of the abnormality. 3,4

HRCT temporal bone is the investigation of choice for evaluating jugular bulb anomalies. ${ }^{4}$

The treatment pattern is mostly conservative and surgical intervention is decided on a case by case basis. However, it is important the surgeon has knowledge of this beforehand to avoid any intraoperative complications during middle ear surgery for any cause.

\section{CONCLUSION}

Jugular Bulb anomalies can present with tinnitus and headache. CT scan is the preferred mode to evaluate these conditions and treatment is generally conservative with surgery reserved for cases with debilitating symptoms.

\section{REFERENCES}

1. Phelps, P.D. and Lloyd, G.A., 1983. Radiology of the Ear. Blackwell Scientific Publications; St. Louis, Mo.: Distributors, USA, Blackwell Mosby.

2. Wadin K, Thomander L, Wilbrand H. Effects of a high jugular fossa and jugular bulb diverticulum on the inner ear. A clinical and radiologic investigation. Acta Radiol Diagn (Stockh) 1986;27(6):629-636.

3. Sasindran V,Joseph A,Abraham SS,Hiremath SB. High riding jugular bulb: A rare entity. Indian J Otol 2014;20(4):129-31.

4. Friedmann DR, Eubig J, Winata LS, Pramanik BK, Merchant SN, Lalwani AK. A Clinical and Histopathologic Study of Jugular Bulb Abnormalities. Arch Otolaryngol Head Neck Surg. 2012;138(1):66-71.

Source of Support: Nil; Conflict of Interest: None

Submitted: 21-01-2020; Accepted: 16-02-2020; Published online: 22-03-2020 